\title{
Focusing on Excessive Gestational Weight Gain through Weight Tracking Among Participants of the Special Supplemental Nutrition Program for Women, Infants, and Children (WIC) in Southern California
}

\author{
Maria Koleilat ${ }^{1}$, Loan P. Kim ${ }^{2}$, and Shannon E. Whaley ${ }^{3}$ \\ ${ }^{1}$ California State University, Fullerton, Department of Health Science \\ ${ }^{2}$ Pepperdine University, Natural Sciences Division \\ ${ }^{3}$ Public Health Foundation Enterprises- Women, Infants and Children (PHFE-WIC)
}

\begin{abstract}
Background and Purpose: Studies have shown that maternal obesity and excessive gestational weight gain (GWG) lead to adverse health outcomes for the mother and her offspring. Yet, knowledge of the best way to intervene is still lacking. The objective of this study was to develop, pilot and evaluate a sustainable intervention to prevent excessive GWG among participants of the Special Supplemental Nutrition Program for Women, Infants, and Children (WIC) in southern California. Methods: We conducted a prospective cohort study where the intervention group received an individual education program that included a GWG tracker. The historical control group was a group of women from the same WIC site who gave birth before the start of the intervention, therefore did not receive the intervention. Chi-square tests and t-tests were used to compare characteristics of the control and intervention groups and the proportions of women exceeding the Institute of Medicine (IOM) recommendations for GWG.

Results: The impact of the intervention was strongest for overweight women. $62.5 \%$ of the control group gained more than the recommended amount compared to $48.6 \%$ in the intervention group. Conclusion: A simple weight tracking intervention could be a cost-neutral way to help WIC participants avoid excessive GWG.
\end{abstract}

(c) 2017 Californian Journal of Health Promotion. All rights reserved.

Keywords: Gestational Weight Gain, WIC, Weight Monitoring, Weight Tracking

\section{Introduction}

Although recent numbers show a slowing or a leveling off of obesity rates in the US, the current high rates of obesity are still a cause of great concern. Recent US data show that, for the years 2013-2014, the overall prevalence of obesity among women was $40.5 \%$ and varied by ethnicity $(12.4 \%$ for non-Hispanic Asians, $38.7 \%$ for non-Hispanic White, $46.6 \%$ for Hispanics, and $57.2 \%$ for non-Hispanic Black) (Flegal, Kruszon-Moran, Carroll, Fryar, \& Ogden, 2016). Similar trends were observed in California. Latinas (33.5\%) and African American (38.9\%) women had higher prevalence of obesity compared to White $(20.6 \%)$ and Asian (8.2\%) women (Wang et al., 2017). Previous studies suggest that social context and cultural factors are possible explanations for these disparities (Bleich,
Thorpe, Sharif-Harris, Fesahazion, LaVeist, 2016; Trotter, Bowen, \& Beresford, 2017).

Women of reproductive age are particularly vulnerable for becoming obese because of excessive gestational weight gain $(\mathrm{GWG})$ and

failing to lose the weight after delivery (Gunderson, Abrams, \& Selvin, 2000; Rooney \& Schauberger, 2002; Smith et al., 1994). Therefore, high gestational weight gain (GWG) along with postpartum weight retention set the stage for suboptimal pre- and perinatal health, increasing the health risks for both mothers and their infants. In response to the evidence, the Institute of Medicine (IOM) revised its 1990 recommendations and released new GWG recommendations in 2009 (Institute of Medicine $[\mathrm{IOM}], 2009)$. The new IOM guidelines are 
specific to a woman's pre-pregnancy Body Mass Index (BMI) and recommend that underweight women (with a pre-pregnancy BMI of $<18.5$ $\mathrm{kg} / \mathrm{m}^{2}$ ) gain $28-40 \mathrm{lbs}$, normal weight women (with a pre-pregnancy BMI of 18.5 - 24.9 $\mathrm{kg} / \mathrm{m}^{2}$ ) gain $25-35 \mathrm{lbs}$, overweight women (with a pre-pregnancy BMI of $25.0-29.9 \mathrm{~kg} / \mathrm{m}^{2}$ ) gain 15-25 lbs, and obese women (with a prepregnancy $\mathrm{BMI}$ of $\geq 30.0$ ) gain $11-20 \mathrm{lbs}$ (Institute of Medicine [IOM], 2009). Despite these guidelines, approximately one-half of women exceeded the recommendations (Deputy, Sharma, \& Kim, 2015), increasing their risks of adverse health outcomes during pregnancy (gestational diabetes, hypertensive disorders, cesarean delivery and operative complications) (Thorsdottir, Torfadottir, Birgisdottir, \& Geirsson, 2002; Cedergren, 2006), and postpartumobesity/overweight (Gunderson, Abrams, \& Selvin, 2000; Rooney \& Schauberger, 2002; Smith et al., 1994; Gunderson \& Abrams, 2000; Rooney, Schauberger, \& Mathiason, 2005). This postpartum period is often the inter-partum period, thus excessive GWG during an earlier pregnancy is a significant nutritional risk factor for subsequent pregnancies. In addition to impacts on mothers, excess GWG can also lead to adverse health outcomes in the offspring, including increased risk of macrosomia and childhood obesity (Thorsdottir, Torfadottir, Birgisdottir, \& Geirsson, 2002; Gillman, RifasShinman, Berkey, Field, \& Colditz, 2003; Olson, Strawdenman, \& Dennison, 2009). Hence, increasing trends of maternal overweight and excessive GWG may create a transgenerational cycle of obesity as heavier mothers give birth to heavier daughters, who are at increased risk of becoming obese themselves during their reproductive years (Dablea \& Crume, 2011). The effect of GWG extends well beyond pregnancy, significantly influencing postpartum health and subsequent pregnancies.

Very little is known about how best to prevent excessive GWG. Findings from previously published studies have been inconsistent with most gestational weight management strategies relying on complex interventions involving intensive diet and physical activity components. Recent reviews and meta-analyses of the literature have concluded that interventions involving diet and physical activity produce only small reductions of GWG (Kuhlmannm, Dietz, Galavotti, \& England, 2008; Streuling, Beyerlein, \& Von Kries, 2010; Dodd, Grivell, Crowther, \& Robinson, 2010; Skouteris et al., 2011; Ronnberg \& Nilsson, 2011; Gardner, Wardle, Poston, \& Croker, 2011). In a systematic review of interventions, while six out of ten studies reported positive intervention effects in relation to GWG, only three studies showed that women in the intervention group were significantly more likely to gain within the IOM guidelines (Skouteris et al., 2010). This systematic review also called for further research to better understand the efficacy of routine pregnancy weighing in predicting excessive GWG and postpartum weight retention. In another review of the literature, Phelan, Jankovitz, Hagobian, \& Abrams (2011) stated that every study that found a positive effect on GWG included weight monitoring as part of the treatment package (Phelan et al., 2011). Weight self-monitoring as part of a lowintensity behavioral intervention was also demonstrated to significantly attenuate GWG in normal weight and class I obese women in a recent study conducted by Krukowski et al. (2017). Furthermore, except for a study conducted by Asbee et al. (2009) and another by Chasan-Taber et al. (2015), most studies to date have been conducted primarily among white individuals (Asbee et al., 2009; Chasan-Taber et al., 2015; Krukowski et al., 2017). Given the known negative consequences of excessive GWG and the scarcity of proven interventions among Hispanic women (Chasan-Taber et al., 2015), a population known for its higher rate of obesity (Robert Wood Johnson Foundation, n.d.), effective interventions among Hispanic women are needed. The purpose of this study was to assess the impact of such an intervention among participants of the WIC program in southern California, a largely Hispanic population.

\section{The Present Study}

Funded by the Food and Nutrition Service (FNS) at the United States Department of Agriculture, this study was designed as a small pilot study to identify the types of intervention 
that may be most successful in the WIC setting. The WIC Program is uniquely positioned to improve prenatal nutrition given its wide reach to the low-income population, who are disproportionately affected by obesity. The WIC Program, created in 1974, is a supplemental nutrition program that provides supplemental foods, breastfeeding support, health care referrals, and nutrition education for low-income pregnant, breastfeeding, non-breastfeeding postpartum women, and to infants and children up to age five who are found to be at nutritional risk. The number of women, infants and children receiving WIC benefits each month was approximately 7.3 million in 2017. Of these, approximately 3.8 million were children, 1.8 million were infants, and 1.7 million were women (United States Department of Agriculture, 2017). As a premier public health nutrition program providing nutritious food and nutrition education supporting pregnant women, infants and young children, WIC plays a critical role in the health of women and children. Strategies found to be successful in impacting the GWG of women on WIC have the potential to significantly impact maternal and child health outcomes.

\section{Intervention}

\section{Methods}

The intervention was designed to help WIC participants avoid gaining weight beyond the IOM recommendations for GWG. Based on extensive qualitative research in which the study team conducted focus groups with pregnant WIC participants (Kim, Koleilat, \& Whaley, 2016), the intervention was designed with two major components that could be easily integrated into core WIC services: guidance about monitoring GWG using a pregnancy weight tracker (GWG Tracker) adapted from the IOM (The Institute of Medicine [IOM] \& National Research Council of the National Academies, n.d.) and accompanying three oneon-one education sessions with a WIC nutritionist.

\section{GWG Monitoring Component Using the GWG Tracker}

The primary intervention material was a pregnancy weight tracker adapted from the IOM
(The Institute of Medicine [IOM] \& National Research Council of the National Academies, n.d.). This tool is used to track weight gain during pregnancy and compare it to the recommended weight ranges for women based on their pre-pregnancy weight. Because weight gain recommendations are not the same for all women, but vary based on their pre-pregnancy weight, dissemination of appropriate materials can be challenging in a busy public health clinic setting. In addition, feedback from focus groups strongly suggested that women prefer information tailored to their specific needs and not generic information that may or may not apply to them (Kim, Koleilat, \& Whaley, 2016). To facilitate ease of use as well as personalization of the tracker, we created trafficlight color-coded trackers for each of the four pre-pregnancy BMI groups: green for normal weight women, yellow for overweight women, red for obese women and blue for underweight women. Distribution of the tracker to each pregnant woman required an assessment of maternal height and pre-pregnancy weight. As this assessment is already part of the WIC standard of care, this step was integrated into existing WIC protocols. To facilitate staff distribution of the appropriate tracker, a "Weight Categories for Women" BMI chart was created (Figure 1) so that staff could easily find the mother's height and pre-pregnancy weight on the chart and immediately identify what color tracker she should receive. The tracker was printed in a small, credit-card sized format and also included in a larger format on the weight gain educational handout described below.

\section{Individual Education Component: GWG Tracker Tool and Education Handout}

An educational four-page handout that contained information about weight gain during pregnancy was designed by the authors based on focus group feedback (Kim, Koleilat, \& Whaley, 2016). Topic areas included: the consequences of excessive GWG, healthy meal ideas using WIC foods, ideas on how to fight cravings, and advice regarding exercise during pregnancy. The GWG tracker was included on the back of the educational handout. The educational handouts used in this study can be found at 
A.
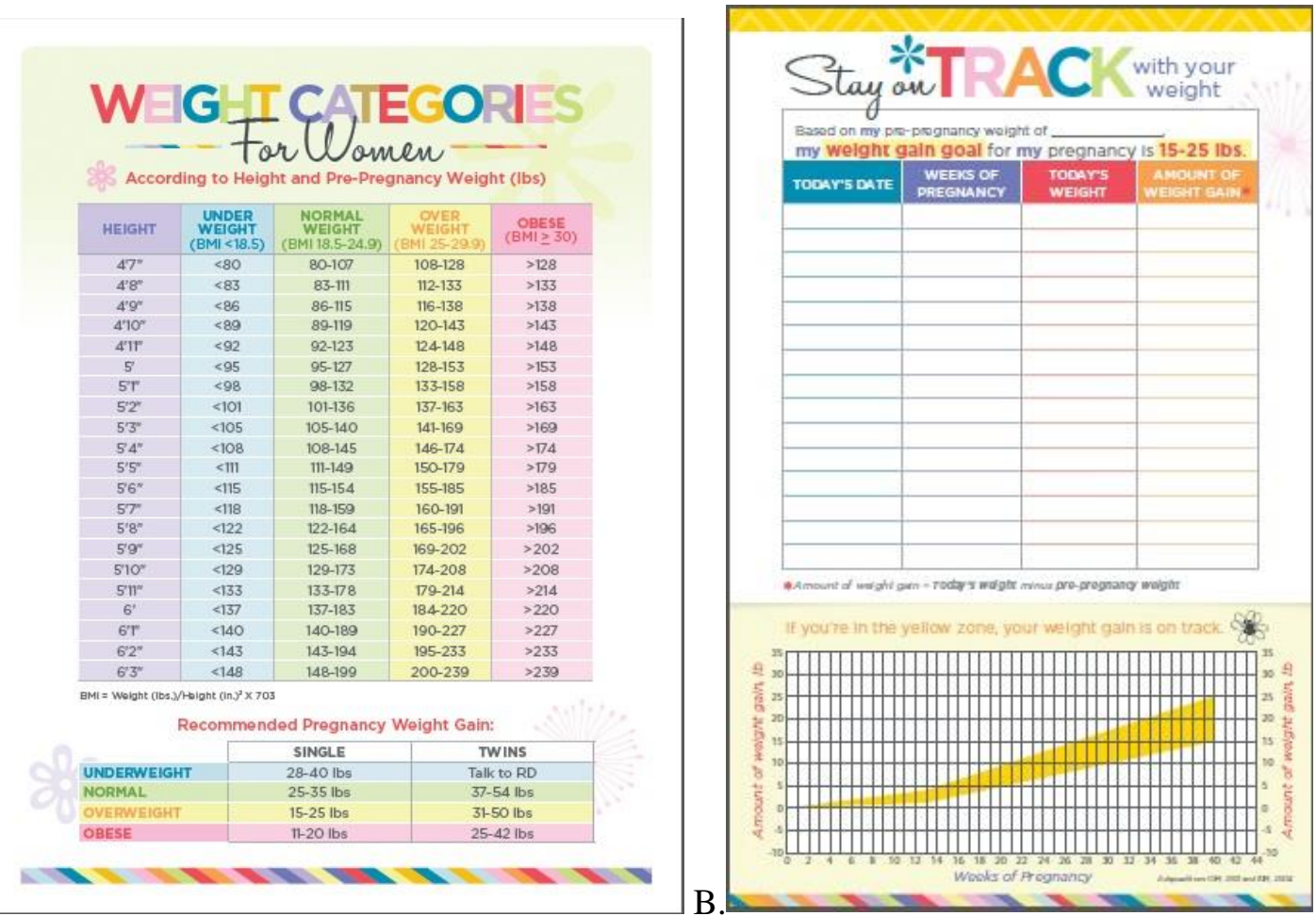

Figure 1 - GWG Tool. (A) The "Weight Categories for Women" BMI chart guides WIC staff to provide appropriate color-coded handouts (B) based on pre-pregnancy BMI ranges. A participant who is 5'3" with a pre-pregnancy weight of $153 \mathrm{lbs}$ falls into the "yellow" (overweight) column in (A). Staff would provide the corresponding "yellow" handout (B) with weight gain goals. If a participant falls in the "blue" (underweight) column, then staff would provide her with the corresponding "blue" handout. If a participant falls in the "green" (normal weight) column, then staff would provide her with the corresponding "green" handout. If a participant falls in the "pink" (obese) column, then staff would provide her with the corresponding "pink" handout. The educational handouts used in this study can be found at http://www.phfewic.org/Projects/GestationalWeightGain.aspx.

\section{Intervention Procedures}

The intervention consisted of three individual contacts with WIC staff, one at each trimester. WIC staff was trained by the authors during two 45-minute orientation sessions and several oneon-one booster sessions. Staff was trained on determining pre-pregnancy BMI, selecting the appropriate tracker based on pre-pregnancy BMI, plotting GWG on the tracker, showing the mother how to track her own weight, and providing healthy weight gain guidance using the weight gain pamphlet. During the WIC enrollment visit, women provided written consent to participate in research and completed the enrollment questionnaire. During this same visit, women were weighed by WIC staff and given the small credit-card sized tracker and the tracker and how to use the tracker. Staff reviewed with the woman the different sections of the pamphlet and answered any questions the woman had. At the end of this first visit, women were encouraged to weigh themselves at home regularly and chart their weight on the tracker. Women were also encouraged to carry the small credit-card sized tracker with them to their obstetrician's appointments and bring it with them to their next WIC visit. At the subsequent visits to WIC in the $2^{\text {nd }}$ and $3^{\text {rd }}$ trimesters, WIC staff weighed the woman, entered the weight on the tracker, answered any questions, and provided guidance to those who were gaining below or above the IOM recommendations. 


\section{Design, Population, and Sample}

Pregnant WIC participants were recruited from one WIC center to enroll in this prospective cohort study that used a historical control group. Recruitment took place between June 2013 and December 2013. The historical control group consisted of a matched group of women from the same WIC site who were pregnant and delivered full term infants in the three months prior to the start of the intervention, therefore did not receive the intervention. We were able to recruit 348 participants in the intervention group and 168 in the control group. However, a total of 222 women from the intervention group and 72 women from the control group were excluded as they had incomplete data. The analytical sample for this study consisted of women from both the control $(n=96)$ and intervention groups $(n=126)$ who enrolled in WIC at first trimester, were 18 years or older, were free of any condition that might impact their body weight, were mentally competent to give informed consent and gave birth to a live, singleton, term infant.

\section{Data Collection and Variables}

Demographic data were collected at enrollment. Data on behavioral variables before and during pregnancy including dietary data were collected at enrollment and at 6-months postpartum via questionnaires that were completed by the participants during their WIC visits. In addition, body weight and height were measured at each of the trimester prenatal WIC visit according to WIC protocol. Data from controls were collected retrospectively during their 6-months postpartum visit. The procedures for this study were approved by the Institutional Review Board of the University of California, Los Angeles and Pepperdine University.

Outcome Variable. The main outcome variable was the proportion of women exceeding the upper limit of the IOM guidelines for GWG that is $40 \mathrm{lbs}$ for underweight women, $35 \mathrm{lbs}$ for those in the normal BMI category, $25 \mathrm{lbs}$ for those in the overweight BMI category, and 20 lbs for those in the obese BMI category (Institute of Medicine [IOM], 2009). Prepregnancy BMI was calculated using measured height and self-reported pre-pregnancy weight. Compliance with IOM weight gain guidelines was determined by comparing the self-reported weight gain during the 6-months postpartum visit with the 2009 IOM guidelines.

\section{Sample Size Justification and Data Analysis}

The intervention study was designed to detect $20 \%$ reduction in the prevalence of excessive GWG in the overall sample at $\alpha=0.05$ using a 2sided test of significance. The proposed final sample size of 118 participants in each group had 95\% statistical power to detect a $20 \%$ reduction in the prevalence of excessive GWG from the observed $24 \%$ gaining above the range in our previous work (Koleilat \& Whaley, 2013). Chi-square tests for categorical variables and ttests for continuous variables were used to compare characteristics of the control and intervention groups. The proportions of women exceeding the IOM recommendations for GWG were compared using $\mathrm{X}^{2}$ test. These comparisons were made for the whole sample and by prepregnancy BMI (normal, overweight, and obese). All analyses were performed using SAS version 9.4 (SAS Institute, Cary, NC).

\section{Results}

Except for the proportion of African-Americans, women in the intervention and control groups did not differ with regards to sociodemographic characteristics (Table 1). In both groups, about $56 \%$ of the women were overweight or obese before pregnancy, around 54\% were married or in a domestic relationship, the majority was Hispanic, their mean age was around 30 and the mean number of years of school completed was around 13 .

In the control group, $55.2 \%$ of women gained more than recommended for their weight category. In the intervention group, $49.6 \%$ gained more than recommended for their weight category. Although this difference did not achieve statistical significance, the impact of the intervention was strongest for overweight women, where $62.5 \%$ of the control group exceeded recommendations compared to $48.6 \%$ of the intervention group. Among obese women, $65.2 \%$ of women in the control group exceeded recommendations compared to $55.9 \%$ of the intervention group (Table 2). This pilot study 
was not powered to examine the impact of the intervention by pre-pregnancy weight status, but preliminary data about overweight and obese women suggest that WIC-based intervention using a weight gain tracker may help overweight and obese women stay on track.

Table 1

Characteristics of Women in Intervention and Control Groups

\begin{tabular}{|c|c|c|c|c|c|}
\hline & \multicolumn{2}{|c|}{$\begin{array}{c}\text { Intervention } \\
(\mathrm{n}=126)\end{array}$} & \multicolumn{2}{|c|}{$\begin{array}{l}\text { Control } \\
(\mathrm{n}=90)\end{array}$} & \multirow[t]{2}{*}{$P$} \\
\hline & $\mathrm{n}$ & $\%$ & $\mathrm{n}$ & $\%$ & \\
\hline Pre-pregnancy & & & & & $0.99^{+}$ \\
\hline BMI & & & & & \\
\hline Underweight & 8 & 6.35 & 6 & 6.67 & \\
\hline Normal & 48 & 38.10 & 35 & 38.89 & \\
\hline Overweight & 35 & 27.78 & 25 & 27.78 & \\
\hline Obese & 35 & 27.78 & 24 & 26.67 & \\
\hline Marital status & 122 & & 89 & & $0.99^{+}$ \\
\hline $\begin{array}{l}\text { Single, never } \\
\text { married }\end{array}$ & 49 & 40.16 & 35 & 39.33 & \\
\hline $\begin{array}{l}\text { Married/domes } \\
\text { tic partnership }\end{array}$ & 65 & 53.28 & 48 & 53.93 & \\
\hline Widowed & 0 & 0 & 0 & 0 & \\
\hline Divorced & 3 & 2.46 & 2 & 2.25 & \\
\hline Separated & 5 & 4.10 & 4 & 4.49 & \\
\hline Ethnicity & 124 & & 88 & & $\begin{array}{r}0.129 \\
++\end{array}$ \\
\hline Hispanic & 87 & 70.16 & 65 & 73.86 & \\
\hline $\begin{array}{l}\text { African- } \\
\text { American }\end{array}$ & 13 & 10.48 & 2 & 2.27 & \\
\hline Asian & 5 & 4.03 & 4 & 4.55 & \\
\hline White & 5 & 4.03 & 5 & 5.68 & \\
\hline American & 0 & 0 & 0 & 0 & \\
\hline Indian & & & & & \\
\hline $\begin{array}{l}\text { Pacific } \\
\text { Islander }\end{array}$ & 1 & 0.81 & 3 & 3.41 & \\
\hline Other & 1.61 & 2 & 4 & 4.55 & \\
\hline Multiracial & 11 & 8.87 & 5 & 5.68 & \\
\hline & $\mathrm{n}$ & $\begin{array}{r}\mathrm{M} \\
(\mathrm{SD})\end{array}$ & $\mathrm{n}$ & $\begin{array}{r}\mathrm{M} \\
(\mathrm{SD})\end{array}$ & \\
\hline Gravidity & 112 & $\begin{array}{r}2.54 \\
(1.67)\end{array}$ & 85 & $\begin{array}{r}2.58 \\
(1.46)\end{array}$ & $\begin{array}{r}0.88 \\
+++\end{array}$ \\
\hline Age in years & 125 & $\begin{array}{r}29.60 \\
(5.8)\end{array}$ & 92 & $\begin{array}{r}30.16 \\
(6.5)\end{array}$ & $\begin{array}{r}0.49 \\
++\end{array}$ \\
\hline $\begin{array}{l}\text { \# years of } \\
\text { school } \\
\text { completed }\end{array}$ & 116 & $\begin{array}{r}12.8 \\
(2.6)\end{array}$ & 84 & $\begin{array}{r}13.2 \\
(3.5)\end{array}$ & $\begin{array}{r}0.36 \\
+++\end{array}$ \\
\hline
\end{tabular}

Table 2

Percent of Women Gaining above IOM Range

\begin{tabular}{llll}
\hline & Intervention & Control & $P$ \\
\hline All & $49.6 \%$ & $55.2 \%$ & 0.43 \\
Normal Weight & $47.9 \%$ & $50 \%$ & 0.85 \\
Overweight & $48.6 \%$ & $62.5 \%$ & 0.29 \\
Obese & $55.9 \%$ & $65.2 \%$ & 0.48 \\
\hline Compliance with IOM weight gain guidelines was \\
determined by comparing the self-reported weight \\
gain during the 6-months postpartum visit with the \\
2009 IOM guidelines.
\end{tabular}

\section{Discussion}

This study is innovative in testing the use of a low-intensity intervention, a pregnancy weight tracker, to help low-income WIC participants avoid excessive GWG. This study was designed to be easily introduced into WIC routine support of pregnant women. The materials developed by this project filled an important gap for WIC nutritionists and paraprofessional staff. GWG is a focus of the WIC program but there are few tools available to facilitate a discussion between WIC staff and mothers about appropriate weight gain. It is a difficult topic primarily because women of different pre-pregnancy weights have different weight gain goals, thus determining the correct guidance to provide can be challenging. The materials developed for this project simplified the assessment of appropriate weight gain and enabled a straightforward conversation about weight gain goals during pregnancy. In addition, the team conducted focus groups with pregnant WIC participants and WIC staff before and during the development of the materials (Kim, Koleilat, \& Whaley, 2016). Direct input from WIC clients and staff ensured that the intervention materials would be optimally suited to WIC program delivery and maximally useful to clients.

Strengths of this study include its low-cost and the use of a low-intensity intervention strategy that can be easily introduced into the WIC routine prenatal care. Another strength is that, although not statistically significant, our conservative intervention reduced excessive GWG among the overweight and obese groups. Given their more restrictive weight gain guidelines, overweight and obese groups have been expected in previous studies to be less 
adherent to the IOM guidelines (Phelan et al., 2011; Polley, Wing, \& Sims, 2002). Those same studies have called for more research to determine how best to prevent excessive GWG among overweight women (Phelan et al., 2011; Polley, Wing, \& Sims, 2002). This finding is important because in our previous research we found that more than two-thirds of mothers entered pregnancy overweight or obese and these mothers were then the most likely to gain excessive weight during pregnancy (Koleilat \& Whaley, 2013). An additional strength of this study is its focus on Hispanic women, a population that has been underrepresented in pregnancy research (Frayne, Burns, Hardt, Rosen, \& Moskowitz, 1996; Durant et al., 2007).

\section{Limitations}

Limitations of this study include the lack of randomization of women to an intervention vs control group. A historical control group design was selected because our past research has shown that once nutritionists in the intervention condition are trained with additional information, it is not feasible for them to withhold it from a random selection of clients. Selecting controls from a different WIC site is problematic in that intervention effects could be due to differences in staff or environment. Thus, comparing historical controls from the same WIC site that had contact with the same staff immediately prior to the intervention holds the setting constant and allows for a true assessment of intervention effects. Another limitation of this study was the use of self-reported pre-pregnancy weight, a common problem throughout pregnancy research. Studies have shown that women with higher BMI are more likely to underreport their weight and therefore underestimate their BMI (C. Gorber, Tremblay, Moher, \& B. Gorber, 2007). However, any misclassification of overweight/obese women as normal weight women that might have occurred would have occurred equally in intervention and control groups. In addition, a limitation of this study is the fact that it was not adequately powered to allow subgroup analyses by prepregnancy BMI and detect statistically significant effects of the intervention. That being said, most of the limitations of this study stem from the fact that this was primarily a pilot study testing the protocol so a larger study can be developed. Finally, while the focus of this study on Hispanic women is considered a strength, in that little is known about GWG and optimal interventions among this population (ChasanTaber et al., 2015), findings may be limited to this group.

The WIC program is uniquely positioned to improve periconceptional nutrition given its wide reach to the low-income population, who are disproportionately affected by obesity. In addition, recent research findings showed that certain subgroups within the WIC population appear to be at a greater risk of excessive GWG and may benefit from preventive interventions (Koleilat \& Whaley, 2013). A study conducted by Koleilat and Whaley (2013) examined trends and predictors of excessive GWG among Hispanic WIC participants in southern California and found that pre-pregnancy weight and language preference were significant predictors of excessive GWG (Koleilat \& Whaley, 2013).

Pregnancy is considered a "teachable moment" as women are highly motivated during this period to improve their health to benefit themselves and their children (Phelan, 2010). Given the wide reach and high quality of height/weight measurements taken by the WIC Program (Crespi, Alfonse, Whaley, \& Wang, 2012), WIC providers have the potential to capitalize upon this teachable moment to influence weight gain during pregnancy and reduce the incidence of overweight and obesity in a substantial proportion of low-income women and children. Through monitoring and giving appropriate advice about GWG, WIC providers have the capacity to alter modifiable risk factors and improve a woman's nutrition and health before a subsequent pregnancy and later in life.

\section{Conclusions}

In summary, although results of this study were not statistically significant, this pilot study based on weight monitoring combined with individual education showed promising trends of lower GWG, particularly among overweight and obese 
mothers. The implication of this study is that a simple weight tracking intervention could be a cost-neutral way to help WIC participants avoid gaining beyond the IOM guidelines during pregnancy. In a larger future study, we plan to confirm the efficacy of this low-cost, lowintensity intervention for preventing excessive GWG in a larger sample of WIC participants.

\section{Acknowledgments}

This study was funded by with a sub-award from UCLA, No. 1920 G QA126 (grant agreement between UCLA and USDA (CFDA No. 10.586)). The authors would like to thank the WIC staff for their help in conducting this project which would not have been possible without all of their support. They would also like to thank WIC participants for their participation and their student research assistants, Nancy Vargas and Sean Bell.

\section{References}

Asbee, S. M., Jenkins, T. R., Butler, J. R., White, J., Elliot, M., \& Rutledge, A. (2009). Preventing excessive weight gain during pregnancy through dietary and lifestyle counseling: A randomized controlled trial. Obstetrics \& Gynecology, 113(2 Pt 1), 305-312.

Bleich, S. N., Thorpe, R. J., Jr., Sharif-Harris, H., Fesahazion, R., LaVeist, T. A (2010). Social context explains race disparities in obesity among women. Journal of Epidemiology and Community Health, 64(5), 465-469. doi: 10.1136/jech.2009.096297.

Cedergren, M. (2006). Effects of GWG and body mass index on obstetric outcome in Sweden. International Journal of Gynaecology \& Obstetrics, 93(3), 269-274. Retrieved from http://dx.doi.org/10.1016/j.ijgo.2006.03.002.

Chasan-Taber, L., Marcus, B., Rosal, M., Tucker, K., Hartman, S., Pekow, P.,... Markenson, G. (2015). Proyecto mamá: A lifestyle intervention in overweight and obese hispanic women: A randomised controlled trial--study protocol. BMC Pregnancy and Childbirth, 15, 157.

Crespi, C., Alfonso, V., Whaley, S., \& Wang, M. (2012). Validity of child anthropometric measurements in the special supplemental nutrition program for women, infants, and children. Pediatric Research, 71(3), 286-292.

Dablea, D., \& Crume, T. (2011). Maternal environment and the transgenerational cycle of obesity and diabetes. Diabetes, 60(7), 1849-1855.

Deputy, N. P., Sharma, A. J., Kim, S.Y. (2015). Gestational weight gain—United States, 2012 and 2013. Morbidity and mortality weekly report, 64(43). Centers for Disease Control and Prevention. Retrieved from http://www.cdc.gov/mmwr/preview/mmwrhtml/mm6443a3.htm.

Dodd, J. M., Grivell, R. M., Crowther, C. A., \& Robinson, J. S. (2010). Antenatal interventions for overweight or obese pregnant women: A systematic review of randomised trials. An International Journal of Obstetrics and Gynaecology, 117(11), 1316-1326. doi: 10.1111/j.14710528.2010.02540.x.

Durant, R., Davis, R., St George, D. M. M., Williams, I. C., Blumenthal, C., \& Corbie-Smith, G. M. (2007). Participation in research studies: Factors associated with failing to meet minority recruitment goals. Annals of Epidemiology, 17(8), 634-42.

Flegal, K.M., Kruszon-Moran, D., Carroll, M.D., Fryar, C.D., Ogden, C.L. (2016). Trends in obesity among adults in the United States, 2005 to 2014. JAMA, 315(21), 2284-2291. doi:10.1001/jama.2016.6458.

Frayne, S. M., Burns, R. B., Hardt, E. J., Rosen, A. K., \& Moskowitz, M. A. (1996). The exclusion of non-English-speaking persons from research. Journal of General Internal Medicine, 11(1), 3943.

Gardner, B., Wardle, J., Poston, L., \& Croker, H. (2011). Changing diet and physical activity to reduce gestational weight gain: a meta-analysis. International Association for the Study of Obesity, 12(7), e602-e620. doi: 10.1111/j.1467-789X.2011.00884.x.

Gillman, M. W., Rifas-Shinman, S., Berkey, C. S., Field, A. E., \& Colditz, G.A. (2003). Maternal gestational diabetes, birth weight, and adolescent obesity. Pediatrics, 111(3), e221-e226. 
Gorber, C. S., Tremblay, M., Moher, D., \& Gorber, B. (2007). A comparison of direct vs. self-report measures for assessing height, weight and body mass index: A systematic review. Obesity Reviews, 8(4), 307-326.

Gunderson, E. P., Abrams, B., \& Selvin, S. (2000). The relative importance of gestational gain and maternal characteristics associated with the risk of becoming overweight after pregnancy. International Journal of Obesity and Related Metabolic Disorders, 24(12), 1660-1668.

Gunderson, E. P., \& Abrams, B. (2000). Epidemiology of gestational weight gain and body weight changes after pregnancy. Epidemiologic Reviews, 22(2), 261-274.

Kim, L. P., Koleilat, M., \& Whaley, S. E. (2016). A qualitative study to examine perceptions and barriers to appropriate gestational weight gain among participants in the Special Supplemental Nutrition Program for Women, Infants and Children (WIC) program. Journal of Pregnancy, vol 2016. doi: http://dx.doi.org/10.1155/2016/4569742.

Koleilat, M., \& Whaley, S. E. (2013). Trends and predictors of excessive gestational weight gain among Hispanic WIC participants in Southern California. Maternal and Child Health Journal, 17(8), 1399-1404.

Krukowski, R.A., West, D., DiCarlo, M., Shankar, K., Cleves, M.A., Tedford, E., Andres, A. (2017). A behavioral intervention to reduce excessive gestational weight gain. Maternal and Child Health Journal, 21,485-491. doi: 10.1007/s10995-016-2127-5.

Kuhlmann, A. K., Dietz, P. M., Galavotti, C., \& England, L. J. (2008). Weight-management interventions for pregnant or postpartum women. American Journal of Preventive Medicine, 34(6), 523-528.

Olson, C. M., Strawdenman, M. S., \& Dennison, B. A. (2009). Maternal weight gain during pregnancy and child weight at age 3 years. Maternal and Child Health Journal, 13(6), 839-846.

Phelan, S. (2010). Pregnancy: a "teachable moment" for weight control and obesity prevention. Obstetrics \& Gynecology, 202(2), 135.e1-135.e8.

Phelan, S., Phipps, M. G., Abrams, B., Darroch, F., Schaffner, A., \& Wing, R. R. (2011). Randomized trial of a behavioral intervention to prevent excessive gestational weight gain: the Fit for delivery study. American Journal of Clinical Nutrition, 93(4), 772-779.

Polley, B. A., Wing, R. R., \& Sims, C. J. (2002). Randomized controlled trial to prevent excessive weight gain in pregnant women. International Journal of Obesity and Related Metabolic Disorders, 26(11), 1494-1502.

Rasmussen, K. M., Yaktine, A. L., \& Institute of Medicine(IOM). (2009). Weight gain in pregnancy: Reexamining the guidelines. Washington, DC: The National Academies Press.

Robert Wood Johnson Foundation. (n.d.). Racial and ethnic disparities in obesity: An in-depth look at the inequities that contribute to higher obesity rates in Black and Latino communities. Retrieved from http://stateofobesity.org/disparities/

Ronnberg, A. K., \& Nilsson, K. (2011). Interventions during pregnancy to reduce excessive gestational weight gain: A systematic review assessing current clinical evidence using the Grading of Recommendations, Assessment, Development and Evaluation (GRADE) system. International Journal of Gynaecology \& Obstetrics, 117(11),1327-1334. doi: 10.1111/j.14710528.2010.02619.x.

Rooney, B. L., \& Schauberger, C. W. (2002). Excess pregnancy weight gain and long-term obesity: One decade later. Obstetrics \& Gynecology, 100(2), 245-252, 2002.

Rooney, B. L., \& Schauberger, C. W., \& Mathiason, M. A. (2005). Impact of perinatal weight change on long-term obesity and obesity-related illnesses. Obstetrics \& Gynecology, 106(6), 1349-1356.

Shirazian, T., Monteith, S., Friedman, F., \& Rebarber, A. (2010). Lifestyles modification program decreases weight gain in obese women. American Journal of Perinatology, 27(5), 411-414.

Skouteris, H., Hartley-Clark, L., McCabe, M., Milgrom, J., Kent, B., et al. (2010). Preventing excessive gestational weight gain: a systematic review of interventions," Obesity Reviews, vol. 11(11), 757768.

Smith, D. E., Lewis, C. E., Caveny, J. L., Perkins, L. L., Burke, G. L., \& Gild, D. E. (1994). Longitudinal changes in adiposity associated with pregnancy. The CARDIA Study. Coronary Artery Risk 
Development in Young Adults Study. The Journal of the American Medical Association, 271(22), 1747-1751.

Streuling, I., Beyerlein. A., \& Von Kries, R. (2010). Can gestational weight gain be modified by increasing physical activity and diet counseling? A meta-analysis of interventional trials. American Journal of Clinical Nutrition, 92(4),678-687. doi: 10.3945/ajen.2010.29363.

The Institute of Medicine (IOM) \& National Research Council of the National Academies. (n.d.). Healthy weight gain during pregnancy. Retrieved from http://resources.iom.edu/Pregnancy/WhatToGain.html\#

Thorsdottir, I., Torfadottir, J. E., Birgisdottir, B. E., \& R.T. Geirsson. (2002). Weight gain in women of normal weight before pregnancy: Complications in pregnancy or delivery and birth outcome. Obstetrics \& Gynecology, 99(5 part 1), 799-806.

Trotter, L. J., Bowen, D. J., \& Beresford, S. A. A. (2010). Testing for racial/ethnic differences in the association between childhood socioeconomic position and adult adiposity. American Journal of Public Health,100(6),1088-1094. doi: 10.2105/AJPH.2009.173492.

United States Department of Agriculture(USDA). (n.d.) The special nutrition program for women, infants and children (WIC Program). Retrieved from https://fnsprod.azureedge.net/sites/default/files/pd/37WIC_Monthly.pdf

Wang, L., Southerland, J., Wang, K., Bailey, B.A., Alamian, A., Stevens, M.A., \&Wang, Y. (2017). Ethnic differences in risk factors for obesity among adults in California, the United States. Journal of Obesity, vol. 2017, Article ID 2427483, 10 pages, 2017. doi:10.1155/2017/2427483

\author{
Author Information \\ *Maria Koleilat \\ California State University, Fullerton \\ Department of Health Science \\ 800 North State College Blvd. \\ Fullerton, CA 92834 \\ Email: mkoleilat@fullerton.edu \\ Office: (657)278-5423 \\ * corresponding author
}

